\title{
ON A THEOREM OF KŌMURA-KOSHI AND OF ANDÔ-ELLIS
}

\author{
YAU-CHUEN WONG
}

ABSTRACT. Komura and Koshi's result, which states that the topology $\mathfrak{I}$ of a nuclear locally convex vector lattice $(E, C, \mathfrak{T})$ is the topology $o\left(E, E^{\prime}\right)$ of uniform convergence on all order-intervals in $E^{\prime}$, is generalized to the case when $(E, C, \mathfrak{T})$ is only a locally solid space. AndôEllis' theorem, concerning the duality of strict $\$$-cones and normality in normed vector spaces, is generalized to the metrizable case.

1. Introduction. By a locally solid space we mean an ordered convex space $(E, C, \mathcal{T}$ ) such that $\mathcal{T}$ admits a neighbourhood base at 0 consisting of convex and solid sets in $E$. Locally solid spaces share a number of important properties with locally convex vector lattices (for example, see Walsh [9] and [10]).

Kömura and Koshi [4] have shown that the topology $\mathcal{T}$ of a nuclear locally convex vector lattice $(E, C, \mathcal{T})$ is the topology $o\left(E, E^{\prime}\right)$ of uniform convergence on all order-intervals in $E^{\prime}$. This result is generalized to the case when $(E, C, \mathcal{T})$ is a locally solid space.

Andô-Ellis' theorem, which states that for a normed space $(E,\|\cdot\|)$ ordered by a complete cone $C$ the normality of $C^{\prime}$ in $\left(E^{\prime},\|\cdot\|\right)$ implies that $C$ be a strict $B$-cone in $(E,\|\cdot\|)$, is one of the important results in the theory of ordered normed spaces. We shall give, in the final section, a generalization on Andô-Ellis' theorem to the metrizable case.

2. A generalization of Kōmura and Koshi's theorem. Let $(E, C, \mathcal{J})$ be a a locally solid space ( $C$ is binormal for $\mathcal{J}$ in the sense of Walsh [9]) whose dual is denoted by $E^{\prime}$, and suppose that $C^{\prime}$ is the dual cone of $C$. Then the weak topology $\sigma\left(E, E^{\prime}\right)$ is locally o-convex (i.e., $C$ is normal in ( $E$, $\left.\sigma\left(E, E^{\prime}\right)\right)$ ), and it is generated by the family $\left\{p_{f}: f \in C^{\prime}\right\}$ of monotone seminorms, where each $p_{f}$ is defined by $p_{f}(x)=|f(x)|(x \in E)$. Suppose $V_{f}=$ $\left\{x \in E: p_{f}(x) \leq 1\right\}$ and $S\left(V_{f}\right)=\bigcup\left\{[-u, u]: u \in V_{f} \cap C\right\}$. Walsh [9, (1.3.7)] has shown that the gauge function of $S\left(V_{f}\right)$, denoted by $p_{f, s}$, is given by

$$
p_{f, S}(x)=\inf \left\{p_{f}(y): y \in C,-y \leq x \leq y\right\}=\inf \{f(y): y \in C,-y \leq x \leq y\}
$$

therefore the family $\left\{p_{f, s}: f \in C^{\prime}\right\}$ of seminorms generates the locally solid

Received by the editors June 17, 1974.

AMS (MOS) subject classifications (1970). Primary 46A40; Secondary 46A15.

Key words and phrases. Solid, order-convex, decomposable, nuclear, base norm, strict -cones, normal cones, open decomposition, locally solid spaces, locally decomposable spaces, locally $o$-convex spaces. 
topology $\sigma_{S}\left(E, E^{\prime}\right)$ associated with $\sigma\left(E, E^{\prime}\right)$. As $p_{f}(u)=p_{f, s}(u)$ for all $u \in$ $C$, it follows that $p_{f}, s$ is additive on $C$.

For any $f \in C^{\prime}$, as the topological dual $E^{\prime}$ of $(E, C, \mathcal{T})$ is a solid subspace of $E^{*}$ (see $[10,(6.5)]$ ), it follows that $[-f, f] \subset E^{\prime}$; we now define

$$
q_{f}(x)=\sup \{g(x):-f \leq g \leq f\} \quad(x \in E) .
$$

It is known from Peressini $\left[6\right.$, p. 130] that $\left\{q_{f}: f \in C^{\prime}\right\}$ generates the topology $o\left(E, E^{\prime}\right)$ of uniform convergence on all order-intervals in $E^{\prime}$.

It is not hard to see that $q_{f}(x) \leq p_{f, s}(x)(x \in E)$. On the other hand, if $g$ is in the polar of $\left\{x \in E: p_{f, S}(x) \leq 1\right\}$, then $|g(u)| \leq p_{f, S}(u)=f(u)(u \in C)$, so $-f \leq g \leq f$, and thus $p_{f, S}(x) \leq q_{f}(x)(x \in E)$. This remark makes the following result clear.

Lemma 1. Let $(E, C, \mathcal{T})$ be a locally solid space. For any $f \in C^{\prime}$, we have $p_{f, S}=q_{f} ;$ consequently, $\sigma_{S}\left(E, E^{\prime}\right)=o\left(E, E^{\prime}\right)$.

Theorem 2. For a nuclear locally solid space $(E, C, \mathcal{J}), \mathcal{J}$ coincides with o(E, $\left.E^{\prime}\right)$.

Proof. This theorem can be deduced from Walsh's result [9, (3.2.5)] and the polar characterization of local decomposability (see $[9,(1.3 .3)]$ or $[10$, (3.10)]); but we present here a somewhat more direct and elementary proof. Let $U$ be any closed, convex, circled $\mathcal{T}_{\text {-neighbourhood of } 0 \text { and suppose that }}$ $p_{U}$ is the gauge of $U$. The nuclearity of $E$ insures that there exists a convex solid $\mathcal{T}$-neighbourhood $V$ of 0 , a sequence $\left(g_{n}\right)$ in the polar $V^{0}$ of $V$, and $\left(\lambda_{n}\right) \in l_{1}$ such that

$$
p_{U}(x) \leq \sum_{n}\left|\lambda_{n} g_{n}(x)\right| \quad(x \in E) .
$$

Since $E^{\prime}$ is a solid subspace of $E^{*}$, by a theorem of Jameson (see [3, (1.7.1)] or $[10,(1.17)]), V^{0}$ is a solid subset of $E^{\prime}$, hence for any $g_{n}$, there exists $f_{n} \in V^{0} \cap C^{\prime}$ such that $-f_{n} \leq g_{n} \leq f_{n}$. Define $f(x)=\Sigma_{n}\left|\lambda_{n}\right| f_{n}(x)(x \in E)$.

Clearly $f$ is a positive continuous linear functional on $E$. On the other hand, the inequality ( 3 ) insures that

$$
P_{U}(x) \leq \inf \{f(y): y \in C,-y \leq x \leq y\}=p_{f, S}(x)(x \in E)
$$

hence $\mathcal{T}$ is coarser than $\sigma_{S}\left(E, E^{\prime}\right)$. As $\sigma_{S}\left(E, E^{\prime}\right)$ is the smallest locally solid topology finer than $\sigma\left(E, E^{\prime}\right)$, we conclude that $\mathcal{T}=\sigma_{S}\left(E, E^{\prime}\right)$, and hence from Lemma 1 that $\mathcal{T}=o\left(E, E^{\prime}\right)$.

The preceding result was proved by Komura and Koshi [4] in the special case when $(E, C, \mathcal{T})$ is a locally convex vector lattice.

A sequence $\left(x_{n}\right)$ in an ordered convex space $(E, C, \mathcal{T})$ is said to be positive if $x_{n} \in C$ for all $n$. Let $(E, C, \mathcal{J}$ ) be a locally solid space and $N$ the set of all natural numbers. We denote by $l^{1}[N, E]$ (resp. $l^{1}(N, E)$ ) the 
space consisting of all absolutely summable (resp. summable) sequences in $E$. It is not hard to see that if $(E, C, \mathcal{J})$ is metrizable, then $l^{1}[N, E]$ is a solid subspace of the (ordered) product space $\left(E^{N}, C^{N}\right)$.

Corollary 3. A metrizable locally solid space $(E, C, \mathcal{J})$ is nuclear if and only if it satisfies the following conditions:

(a) $\mathfrak{T}=o\left(E, E^{\prime}\right)$;

(b) each summable sequence in $E$ is the difference of two positive summable sequences in $E$.

Proof. Suppose that $E$ is nuclear. Then $\mathcal{T}=o\left(E, E^{\prime}\right)$ by Theorem 2, and $l^{1}(N, E)=l^{1}[N, E]$ in view of a well-known result. According to the remark preceding the corollary, $l^{1}[N, E]$ is a solid subspace of $\left(E^{N}, C^{N}\right)$, hence ( $b$ ) holds; therefore the condition is necessary. To see that it is also sufficient, we first note that each positive summable sequence in $(E, C$, $\left.o\left(E, E^{\prime}\right)\right)$ is absolutely summable with respect to the topology o(E, $\left.E^{\prime}\right)$. Therefore $l^{1}(N, E)=l^{1}[N, E]$. As $E$ is metrizable, we conclude from Pietsch $[8,(4.2 .5)]$ that $E$ is nuclear.

For a base normed space $(E, C,\|\cdot\|)$, the norm $\|\cdot\|$ is additive on $C$, it follows from Walsh $[9,(3.2 .5)]$ that the norm topology coincides with o(E, $\left.E^{\prime}\right)$. But a normed space with infinite dimension is not nuclear, therefore condition (b) in Corollary 3 is essential.

3. A generailzation of Ando-Ellis' theorem. For a subset $V$ of $(E, C)$, we define

$$
D(V)=\{x \in V: x=\lambda u-(1-\lambda) w, \lambda \in[0,1] \text { and } u, w \in V \cap C\} \text {. }
$$

It is clear that if $V$ is convex and circled, then $D(V)=\operatorname{co}\{-(V \cap C) \cup(V \cap C)\}$ and

$$
(V \cap C-V \cap C) / 2 \subset D(V) \subset V \cap C-V \cap C .
$$

A subset $V$ of $(E, C)$ is said to be decomposable if $V=D(V)$. An ordered convex space $(E, C, \mathcal{T}$ ) is called a locally decomposable space ( $C$ is conormal in $(E, \mathcal{T})$ in the sense of Walsh [9]) if $\mathcal{T}$ admits a neighbourhood base at 0 consisting of convex decomposable sets in $E$. According to formula (4), an ordered convex space $(E, C, \mathcal{T})$ is locally decomposable if and only if $C$ gives an open decomposition in $(E, \mathfrak{T})$ in the sense of Jameson [3, p. 94]. The topological dual $E^{\prime}$ of a locally decomposable space $(E, C, \mathcal{T})$ is an order-convex subspace of $E^{*}$.

In this section $\beta\left(E^{\prime}, E\right)$ denotes the strong topology on $E^{\prime}$ while $\sigma\left(E^{\prime}, E\right)$ is the weak topology on $E^{\prime}$.

Theorem 4. Let $(E, C, \mathcal{T})$ be an infrabarrelled ordered convex space with a countable fundamental system of $\mathcal{T}$-bounded subsets of $E$. If $(E, C, \mathcal{T})$ 
is locally decomposable then $\left(E^{\prime}, C^{\prime}, \beta\left(E^{\prime}, E\right)\right.$ ) is locally o-convex.

Proof. According to the hypotheses, $\left(E^{\prime}, C^{\prime}, \beta\left(E^{\prime}, E\right)\right)$ is metrizable, therefore it is sufficient to show, by $[10,(5.2)]$, that the order-convex hull (in $E^{\prime}$ ) of each $\beta\left(E^{\prime}, E\right)$-bounded subset $B$ of $E^{\prime}$ is $\beta\left(E^{\prime}, E\right)$-bounded. Let $B$ be such a set. $B$ is $\mathcal{T}$-equicontinuous, it follows from the polar characterization of local decomposability (see Walsh $[9,(1.3 .3)]$ or $[10,(3.10)]$ ) that $\left(B+C^{*}\right) \cap\left(B-C^{*}\right)$ is $\mathcal{T}$-equicontinuous; consequently, the order-convex hull of $B$ is $\beta\left(E^{\prime}, E\right)$-bounded.

Corollary 5. Let $(E, C, \mathcal{T})$ be an infrabarrelled ordered convex space with a countable fundamental system of bounded sets. If $(E, C, \mathcal{T})$ is locally solid then so is $\left(E^{\prime}, C^{\prime}, \beta\left(E^{\prime}, E\right)\right.$ ).

Proof. It is known from $[10,(6.3)]$ that an ordered convex space is locally solid if and only if it is both locally o-convex and locally decomposable Now Theorem 4 insures that $\left(E^{\prime}, C^{\prime}, \beta\left(E^{\prime}, E\right)\right.$ ) is locally o-convex. On the other hand, by Schaefer's theorem [8, p. 220], the local o-convexity of $\mathfrak{T}$ implies that $C^{\prime}$ is a strict $\mathcal{B}$-cone in $\left(E^{\prime}, \beta\left(E^{\prime}, E\right)\right.$ ); by Jameson [3, (3.3.6)] (or $[9,(1.2 .4)]),\left(E^{\prime}, C^{\prime}, \beta\left(E^{\prime}, E\right)\right)$ is locally decomposable because $\left(E^{\prime}\right.$, $\left.\beta\left(E^{\prime}, E\right)\right)$ is metrizable and surely bomological.

The following result should be compared with Jameson [3, (3.5.4)].

Theorem 6. Let $(E, C, \mathcal{T})$ be a metrizable ordered convex space such that $C$ is $\mathcal{T}_{\text {-complete. If }}\left(E^{\prime}, C^{\prime}, \beta\left(E^{\prime}, E\right)\right.$ ) is locally o-convex then $(E$, $C, \mathcal{T}$ ) is locally decomposable, and hence $E$ is $\mathcal{T}$-complete.

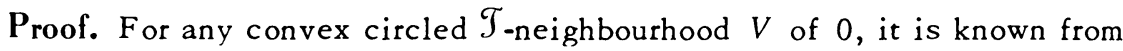
$[10,(2.11)]$ that $(D(V))^{0}=\left(V^{0}+C^{*}\right) \cap\left(V^{0}-C^{*}\right) \cap E^{\prime}$, therefore $(D(V))^{0}$ is the order-convex hull in $E^{\prime}$ of $V^{0}$. As $E$ is metrizable and surely infrabarrelled, it follows from the local o-convexity of $\left(E^{\prime}, C^{\prime}, \beta\left(E^{\prime}, E\right)\right.$ ) that

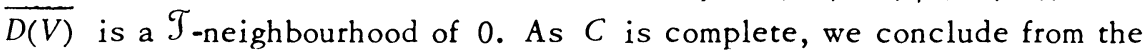
open mapping theorem (see $\mathrm{Ng}[5$, Theorem 2]) that $(E, C, \mathcal{T})$ is locally decomposable.

Finally, since $(E, C, \mathcal{T})$ is locally decomposable for which $C$ is $\mathcal{T}$. complete, it follows from Klee's theoerm (see [10, (3.6)]) that $E$ must be complete. Therefore the proof is complete.

The preceding theorem, together with a result of Schaefer [8, p. 220], shows that for a metrizable ordered convex space $(E, C, \mathcal{T})$ such that $C$ is T-complete, if $\beta\left(E^{\prime}, E\right)$ is locally o-convex then so is $\sigma\left(E^{\prime}, E\right)$.

Clearly an ordered normed space $(E, C,\|\cdot\|)$ is locally decomposable if and only if $C$ is a strict $B$-cone in $(E,\|\cdot\|)$. Therefore Theorems 4 and 6 constitute a generalization of Andô-Ellis' theorem [1], [2] and [5, Theorem 3].

I am very grateful to the referee for many helpful comments. 


\section{REFERENCES}

1. T. Andô, On fundamental properties of a Banach space with a cone, Pacific J. Math. 12 (1962), 1163-1169. MR 27 \#68.

2. A. J. Ellis, The duality of partially ordered normed linear spaces, J. London Math. Soc. 39 (1964), 730-744. MR 29 \#6275.

3. G. J. O. Jameson, Ordered linear spaces, Lecture Notes in Math., vol. 104, Springer-Verlag, Berlin, 1970.

4. Y. Komura and S. Koshi, Nuclear vector lattices, Math. Ann. 163 (1966), 105-110. MR $33 \# 553$.

5. Kung-Fu Ng, An open mapping theorem, Proc. Cambridge Philos. Soc. 74 (1973), 61-66.

6. A. L. Peressini, Ordered topological vector spaces, Harper \& Row, New York, 1967. MR 37 \#3315.

7. A. Pietsch, Nuclear locally convex spaces, Springer-Verlag, Berlin, 1972.

8. H. H. Schaefer, Topological vector spaces, Macmillan, New York, 1966. MR 33 \# 1689.

9. B. Walsh, Ordered vector sequence spaces and related classes of linear operators, Math. Ann. 206 (1973), 89-138.

10. Yau-Chuen Wong and Kung-Fu Ng, Partially ordered topological vector spaces, Oxford Math. Monographs, Clarendon Press, Oxford, 1973.

DEPARTMENT OF MATHEMATICS, YALE UNIVERSITY, NEW HAVEN, CONNECTICUT 06520

UNITED COlLEGE, THE CHINESE UNIVERSITY OF HONG KONG, HONG KONG 